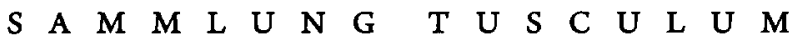

In Tusculum, vor den Toren

Roms, hatte Cicero sein Land-

haus. In Zeiten der Muße, aber

auch der politischen Isolation

zog er sich dorthin zurück.

Tusculum wurde zum Inbegriff

für Refugium, für Muße, für

wertvolle Fluchten aus einem

fordernden Alltag.

In der ersten Phase des Rück-

zugs aus der Politik schrieb

Cicero in Tusculum die

so genannten Tuskulanen,

eine lateinische Einführung in

die Welt der (griechischen)

Philosophie.

Wissenschaftliche Beratung

Niklas Holzberg,

Rainer Nickel,

Karl-Wilhelm Weeber,

Bernhard Zimmermann 

EURIPIDES | AUSGEWÄHLTE TRAGÖDIEN

in zwei Bänden

Griechisch und deutsch

\section{Band II}

Aus dem Griechischen von

Dietrich Ebener

Herausgegeben von

Bernhard Zimmermann 
Die vorliegende Übersetzung von

Dietrich Ebener basiert auf

EURIPIDES: Werke in drei Bänden.

Hrsg., a. d. Griech. u. Anm.

Dietrich Ebener;

Red. Herbert Greiner-Mai;

2. Aufl. 1979 Bibliothek der Antike;

Aufbau-Verlag Berlin und Weimar.

Die Orthographie wurde behutsam auf die neue deutsche Rechtschreibung von 2006 umgestellt.

Bibliographische Information der

Deutschen Nationalbibliothek:

Die Deutsche Nationalbibliothek

verzeichnet diese Publikation

in der Deutschen National-

bibliographie;

detaillierte bibliographische Daten

sind im Internet über

http://dnb.d-nb.de abrufbar.

(C) 2010 Patmos Verlag GmbH \& Co. KG

Artemis \& Winkler Verlag, Mannheim

(C) 1979 Aufbau Verlag GmbH \& Co. KG,

Berlin (für die Übersetzung)

Mit dem Erwerb dieser Ausgabe ist

kein Erwerb von Aufführungsrechten

verbunden.

Alle Rechte vorbehalten.

Printed in Germany

ISBN: 978-3-538-03539-3

www.artemisundwinklerde 